ESAIM: COCV 27 (2021) 20

https://doi.org/10.1051/cocv/2021021
ESAIM: Control, Optimisation and Calculus of Variations

www.esaim-cocv.org

\title{
ROBUST LINEAR QUADRATIC MEAN FIELD SOCIAL CONTROL: A DIRECT APPROACH*
}

\author{
Tinghan Xie ${ }^{1}$, Bing-Chang Wang ${ }^{2, * *}$ And Jianhui Huang ${ }^{1}$
}

\begin{abstract}
This paper investigates a robust linear quadratic mean field team control problem. The model involves a global uncertainty drift which is common for a large number of weakly-coupled interactive agents. All agents treat the uncertainty as an adversarial agent to obtain a "worst case" disturbance. The direct approach is applied to solve the robust social control problem, where the state weight is allowed to be indefinite. Using variational analysis, we first obtain a set of forward-backward stochastic differential equations (FBSDEs) and the centralized controls which contain the population state average. Then the decentralized feedback-type controls are designed by mean field heuristics. Finally, the relevant asymptotically social optimality is further proved under proper conditions.
\end{abstract}

Mathematics Subject Classification. 49N10, 49N70, 91A12, 93E03.

Received December 7, 2020. Accepted February 12, 2021.

\section{INTRODUCTION}

The mean field game (MFG) [9, 34], a very powerful tool in solving problems with large-population characteristic, is applied in many fields such as information technology and engineering [15, 28, 31], crowd motion $[1,8,33]$, finance and economics $[13,19,47,49]$, vaccination and medicine $[5,12,21,24,35]$, especially for the following recent papers connected to the COVID-19 pandemic $[2,14,17]$. The mean field optimal control problem, a special class of control problems, is introduce in $[6,11]$. One special case of the mean field optimal control problem is the linear quadratic mean field (LQ-MF) control, which can model many problems in applications like $[3,10,26,36,43]$.

Social optimization (team optimization) problem is a joint decision problem in which all the agents have the same goal and work collaboratively to optimize the social cost by picking their optimal strategies. Ho and Chu studied optimal control problems under team decision theory in [23]. In [29], Huang investigated the social optima in LQ-MF control problems with centralized and decentralized strategies, which was further generalized to the case with Markov jump parameters [44]. The team problems under stochastic information structure with

*The first author acknowledges the financial support from: P0008686, P0031044. The second author acknowledges the support from: NNSF of China (61773241), the Youth Innovation Group Project of Shandong University (2020QNQT016). The third author acknowledges the support from: RGC 153005/14P, 153275/16P, P0030808. The authors also acknowledge the support from: The PolyU-SDU Joint Research Centre on Financial Mathematics.

Keywords and phrases: Mean field game, model uncertainty, linear quadratic control, social optimality, forward-backward stochastic differential equation.

1 Department of Applied Mathematics, The Hong Kong Polytechnic University, Hong Kong, PR China.

2 School of Control Science and Engineering, Shandong University, Jinan, PR China.

** Corresponding author: bcwang@sdu.edu.cn 
suboptimal solutions was studied in [22] and the social functional variation and person-by-person optimality was exploiting in [46].

In general, mathematical models only describe and simulate the complicatedly real world in an approximated approach. Therefore, it is very meaningful to investigate a model with uncertainty terms [4]. In [25, 26, 45, 46], the mean field LQ control problem with a global uncertainty term is considered by researchers. More details, in [25], the so called "hard constraint" approach was adopted to overcome difficulties after using the Lagrange multiplier. The "soft constraint" case (see $[4,7,18]$ ) was investigated in $[26,45,46]$, which removed the bound of the disturbance and add a penalty for the disturbance in cost functional. The situation that a local disturbance appears between each agent was studied in [38, 42].

This paper studies the social optimality of the robust LQ-MF control model with a common uncertain drift by using a direct approach. The common uncertain term appears in both state equation and cost functional of each agent. Different from $[26,46]$, the weight coefficient $Q$ of state in the cost functional is allowed to be indefinite. The coefficients of our model are time-varying, which means the coefficients can be changed at different times. The time-variant systems could be applied in many areas such as earth's thermodynamic and the human vocal tract (see $[39,41])$.

Normally, there are two routes to solve MFG problems. One is called the fixed point approach (see [26, 28, 29, 46]), which starts by applying mean field approximation and constructing a fixed point problem. Then, the $N$-player game is degenerated to an optimal control problem. By analyzing the optimal response of the representative player, the decentralized strategies can be designed, which are proved are asymptotically optimal. Another route is called the direct approach (see [16,30,34,48]), which starts by solving the $N$-player game problem formally under a large population and high dimensional environment. Then, by letting $N$ goes to infinite, one can have the decentralized optimal control.

In this paper, an LQ-MF team problem with a common uncertain drift is solved by using a direct approach. We first perturb all the agents and used duality procedures to tackle the large-scale problem with high dimensional FBSDE. After that, the centralized controls explicitly depending on $x_{i}$ and the state average $x^{(N)}$ are obtained first and then the decentralized controls are designed by mean field heuristics. Compared with previous works, this paper mainly makes the following contributions:

- Instead of using the fixed-point method (see [45, 46]), the direct approach is applied to solve the robust LQ-MF social control problem, where the state weight $Q$ is allowed to be indefinite.

- By the solvability of low-dimensional Ricatti-liked equation system, we obtain the condition for the uniform convexity of a high-dimensional control problem (P3).

- Comparing to $[45,46]$ whose consistency condition (CC) system contains five coupled equations, we just have four coupled Ricatti-liked equations, which is much easier to tackle. The number of coupled equations can be even reduced to three under a specific condition. Moreover, in proving asymptotic optimality, we obtain the consistency of mean-field approximations without setting an additional assumption.

The paper is organized as follows. The robust LQ-MF problem is formulated in Section 2. In Section 3, we solve the worst disturbance based on the maximum principle. In Section 4, we seek the social optimal solution under the "worst case" uncertainty and design asymptotically optimal decentralized controls by handling coupled FBSDEs. In Section 5, we prove the asymptotically social optimality of decentralized controls. In Section 6, a numerical example is provided to simulate the efficiency of decentralized control. Section 7 is the conclusion of the paper.

Notation: Throughout this paper, $\mathbb{R}^{n \times m}$ and $\mathbb{S}^{n}$ denote the set of all $(n \times m)$ real matrices and the set of all $(n \times n)$ symmetric matrices, respectively. $|\cdot|$ is the standard Euclidean norm and $\langle\cdot, \cdot\rangle$ is the standard Euclidean inner product. For given symmetric matrix $S \geq 0$, the quadratic form $x^{T} S x$ may be defined as $|x|_{S}^{2}$, where $x^{T}$ is the transpose of $x . C^{1}\left([0, T] ; \mathbb{R}^{n \times m}\right)$ be the space of all $\mathbb{R}^{n \times m}$-valued continuously differentiable functions on $[0, T]$. For notation $o(1), \lim _{n \rightarrow \infty} o(1)=0$ and $\bigvee_{\alpha}:=\sigma\left(\bigcup_{\alpha} \mathcal{F}_{\alpha}\right)$. For the sake of notation simplicity, we will use $c$ to denote a generic constant in following discussion. The value of $c$ may be different at different places and it only depends on the coefficients and initial values. 


\section{Problem Formulation}

Let $\left(\Omega, \mathcal{F},\left\{\mathcal{F}_{t}\right\}_{0 \leq t \leq T}, \mathbb{P}\right)$ be a complete filtered probability space augmented by all $\mathbb{P}$-null sets in $\mathcal{F}$. $W_{i}(\cdot)$, $i=1, \ldots, N$ are independent, $d$-dimensional standard Brownian motions defined on $(\Omega, \mathcal{F}, \mathbb{P})$. The initial states values $\xi_{i} \in \mathbb{R}^{n}$. We define $\sigma$-algebra $\mathcal{F}_{t}^{i}=\sigma\left(W_{i}(s), 0 \leq s \leq t\right)$ and $\mathcal{G}_{t}^{i}=\mathcal{F}_{t}^{i} \bigvee \sigma\left\{\xi_{i}\right\}$, where $1 \leq i \leq N$. $\mathcal{F}_{t}=\sigma\left(W_{i}(s), 0 \leq s \leq t, 1 \leq i \leq N\right)$ and $\mathcal{G}_{t}=\mathcal{F}_{t} \bigvee \sigma\left\{\xi_{i}, 1 \leq i \leq N\right\} . \mathbb{F}^{i}=\left\{\mathcal{F}_{t}^{i}\right\}_{0 \leq t \leq T}$ is the natural filtration generated by $W_{i}(\cdot)$ and $\mathbb{G}^{i}=\left\{\mathcal{G}_{t}^{i}\right\}_{0 \leq t \leq T}$, where $1 \leq i \leq N$. Correspondingly, we denote $\mathbb{F}=\left\{\mathcal{F}_{t}\right\}_{0 \leq t \leq T}$, $\mathbb{G}=\left\{\mathcal{G}_{t}\right\}_{0 \leq t \leq T}$. Next we introduce the following spaces:

$$
\begin{gathered}
L_{\mathcal{F}_{T}}^{2}\left(\Omega ; \mathbb{R}^{m}\right)=\left\{\xi: \Omega \rightarrow \mathbb{R}^{m} \mid \xi \text { is } \mathcal{F}_{T^{-}} \text {-measurable, } \mathbb{E}|\xi|^{2}<\infty\right\}, \\
L^{\infty}\left(0, T ; \mathbb{R}^{n \times m}\right)=\left\{\varphi:[0, T] \rightarrow \mathbb{R}^{n \times m} \mid \varphi(\cdot) \text { is bounded and measurable }\right\}, \\
L_{\mathbb{F}}^{2}\left(0, T ; \mathbb{R}^{m}\right)=\left\{x:[0, T] \times \Omega \rightarrow \mathbb{R}^{m} \mid x(\cdot) \text { is } \mathbb{F}\right. \text {-progressively measurable, } \\
\left.\|x\|_{L^{2}}^{2}:=\mathbb{E} \int_{0}^{T}|x(t)|^{2} \mathrm{~d} t<\infty\right\}, \\
L_{\mathbb{F}}^{2}\left(\Omega ; C\left([0, T] ; \mathbb{R}^{m}\right)\right)=\left\{x:[0, T] \times \Omega \rightarrow \mathbb{R}^{m} \mid x(\cdot) \text { is } \mathbb{F}\right. \text {-progressively measurable, } \\
\text { continuous, } \left.\mathbb{E} \sup _{t \in[0, T]}|x(t)|^{2}<\infty\right\} .
\end{gathered}
$$

We consider a large-population system which contains $N$ agents. The state processes of the $i$ th agent, $i=$ $1,2, \ldots, N$, is modelled by the following linear stochastic differential equations (SDE) on a finite time horizon $[0, T]:$

$$
\mathrm{d} x_{i}(t)=\left[A(t) x_{i}(t)+B(t) u_{i}(t)+F(t) x^{(N)}(t)+f(t)\right] \mathrm{d} t+\sigma(t) \mathrm{d} W_{i}(t), \quad x_{i}(0)=\xi_{i}
$$

where $x^{(N)}(t) \triangleq \frac{1}{N} \sum_{i=1}^{N} x_{i}(t)$ is the state average of the agents. $A(\cdot), B(\cdot), F(\cdot), \sigma(\cdot)$ are deterministic matrixvalued functions of suitable sizes. $f(\cdot)$ is an unknown disturbance that agents are imposed by the environment. The coefficients appearing in (2.1) satisfy

(A1)

$$
A(\cdot), F(\cdot), \sigma(\cdot) \in L^{\infty}\left(0, T ; \mathbb{R}^{n \times n}\right), \quad B(\cdot) \in L^{\infty}\left(0, T ; \mathbb{R}^{n \times m}\right), \quad f(\cdot) \in L_{\mathbb{G}}^{2}\left(0, T ; \mathbb{R}^{n}\right) .
$$

The cost functional of the $i$ th agent is given by

$$
\begin{aligned}
\mathcal{J}_{i}^{F}(u(\cdot), f(\cdot))= & \frac{1}{2} \mathbb{E} \int_{0}^{T}\left\{\left|x_{i}(t)-\Gamma(t) x^{(N)}(t)-\eta(t)\right|_{Q(t)}^{2}+\left|u_{i}(t)\right|_{R_{1}(t)}^{2}-|f(t)|_{R_{2}(t)}^{2}\right\} \mathrm{d} t \\
& +\frac{1}{2} \mathbb{E}\left|x_{i}(T)-\hat{\Gamma} x^{(N)}(T)-\hat{\eta}\right|_{G}^{2},
\end{aligned}
$$

where $u(\cdot)=\left\{u_{1}(\cdot), \ldots, u_{N}(\cdot)\right\} \cdot Q(\cdot), R_{1}(\cdot), R_{2}(\cdot)$ and $G$ are weight matrices and the coefficients appearing in (2.2) satisfy

$$
\begin{cases}Q(\cdot) \in L^{\infty}\left(0, T ; \mathbb{S}^{n}\right), & R_{1}(\cdot), R_{2}(\cdot) \in L^{\infty}\left(0, T ; \mathbb{S}^{m}\right), \quad \Gamma(\cdot) \in L^{\infty}\left(0, T ; \mathbb{R}^{n \times n}\right), \\ G \in \mathbb{S}^{n}, \quad \hat{\Gamma} \in \mathbb{R}^{n \times n}, \quad \eta(\cdot) \in L_{\mathbb{F}}^{2}\left(0, T ; \mathbb{R}^{n}\right), \quad \hat{\eta} \in L_{\mathcal{F}_{T}}^{2}\left(\Omega ; \mathbb{R}^{n}\right) .\end{cases}
$$


All the agents in the system work cooperatively to optimize the social cost functional

$$
\mathcal{J}_{\text {soc }}^{F}(u(\cdot), f(\cdot))=\sum_{i=1}^{N} \mathcal{J}_{i}^{F}(u(\cdot), f(\cdot)) .
$$

The decentralized control set is defined as follows:

$$
\mathcal{U}_{i}^{F}=\left\{u_{i} \mid u_{i}(t) \in L_{\mathbb{G}^{i}}^{2}\left(0, T ; \mathbb{R}^{m}\right), 1 \leq i \leq N\right\},
$$

and the decentralized control set of all agents is defined as $\mathcal{U}^{F}=\mathcal{U}_{1}^{F} \times \mathcal{U}_{2}^{F} \times \cdots \times \mathcal{U}_{N}^{F}$. For comparison, the centralized control set is given by

$$
\mathcal{U}_{c}^{F}=\left\{\left(u_{1}, \ldots, u_{N}\right) \mid u_{i}(t) \in L_{\mathbb{G}}^{2}\left(0, T ; \mathbb{R}^{m}\right), 0 \leq i \leq N\right\}
$$

According to the minimax control problem, we need to consider the possible of worst case scenario. Thus, the social cost under the worst-case disturbance as

$$
\mathcal{J}_{\text {soc }}^{w o}(u(\cdot))=\sup _{f \in \mathcal{U}_{c}^{F}} \mathcal{J}_{\text {soc }}^{F}(u(\cdot), f(\cdot)) .
$$

For further analysis, we introduce the following assumptions.

(A3) $\left\{x_{i}(0)\right\}$ are independent with the same expectation. $\mathbb{E} x_{i}(0)=\hat{\xi}, 1 \leq i \leq N$. There exists a constant $c_{0}$ such that $\sup _{1 \leq i \leq N} \mathbb{E}\left|x_{i}(0)\right|^{2} \leq c_{0}$, where $c_{0}$ is independent of $N$. Furthermore, $\left\{x_{i}(0)\right\}$ and $W_{i}(t), i=1,2, \ldots, N$ are mutually independent.

(A4) $R_{1}(\cdot)>0, R_{2}(\cdot)>0$ and $G \geq 0$.

From now on, we may suppress the notation of time $t$ if necessary. Now, we introduce our robust LQ-MF problem:

Problem 2.1. (P1) Seek a set of decentralized control laws $\bar{u}=\left\{\bar{u}_{1}, \ldots, \bar{u}_{N}\right\} \in \mathcal{U}^{F}$ such that for $\varepsilon>0$,

$$
\mathcal{J}_{\text {soc }}^{w o}(\bar{u})-\varepsilon \leq \inf _{u \in \mathcal{U}_{c}^{F}} \mathcal{J}_{\text {soc }}^{w o}(u) \leq \mathcal{J}_{\text {soc }}^{w o}(\bar{u})
$$

\section{Mean field LQ control Problem for the disturbance}

In this section, we seek the worst-case disturbance $f$. First, we fix $u_{i}=\check{u}_{i} \in \mathcal{U}_{c}^{F}, i=1, \ldots, N$ and consider the optimal control problem for the disturbance:

$$
\text { (P2) } \operatorname{maximize}_{f \in \mathcal{U}_{c}^{F}} \mathcal{J}_{\text {soc }}^{F}(\check{u}, f) \text {. }
$$

Then, (P2) can be rewritten as an equivalent problem

$$
\text { (P2a) minimize } \operatorname{f\in \mathcal {U}}_{c}^{F} \check{\mathcal{J}}_{\text {soc }}^{F}(f)
$$

where

$$
\check{\mathcal{J}}_{\text {soc }}^{F}(f)=\frac{1}{2} \sum_{i=1}^{N} \mathbb{E} \int_{0}^{T}\left\{-\left|x_{i}-\Gamma x^{(N)}-\eta\right|_{Q}^{2}+|f|_{R_{2}}^{2}\right\} \mathrm{d} t-\frac{1}{2} \sum_{i=1}^{N} \mathbb{E}\left|x_{i}(T)-\hat{\Gamma} x^{(N)}(T)-\hat{\eta}\right|_{G}^{2} .
$$

Here $x_{i}$ are the solution to corresponding $\check{u}_{i}, i=1,2, \ldots, N$. To obtain the worst disturbance, we need to discuss the convexity of (3.1). 
Let $\mathbf{x}=\left(x_{1}, \ldots, x_{N}\right)^{T}, \mathbf{u}=\left(u_{1}, \ldots, u_{N}\right)^{T}, \mathbf{W}=\left(W_{1}, \ldots, W_{N}\right)^{T}, \mathbf{A}=\operatorname{diag}(A, \ldots, A), \mathbf{B}=\operatorname{diag}(B, \ldots, B)$ and $\check{\sigma}=$ $\operatorname{diag}(\sigma, \ldots, \sigma)$. Then our state equation can be rewritten as

$$
d \mathbf{x}=(\check{\mathbf{A}} \mathbf{x}+\mathbf{B u}+\mathbf{1} \otimes f) \mathrm{d} t+\check{\sigma} d \mathbf{W}(t),
$$

where $\check{\mathbf{A}}=\mathbf{A}+\frac{1}{N}\left(\mathbf{1 1}^{T} \otimes F\right), \mathbf{1}=(1, \ldots, 1)^{T}$. Correspondingly, the Problem $(\mathbf{P} 2 \mathbf{a})$ can be rewritten as

$$
\min _{f \in \mathcal{U}_{c}^{F}}\left\{\frac{1}{2} \mathbb{E} \int_{0}^{T}\left(-\mathbf{x}^{T} \mathbf{Q} \mathbf{x}+2 \check{\eta} \mathbf{x}+N f^{T} R_{2} f\right) \mathrm{d} t-\frac{1}{2} \mathbb{E}\left[\mathbf{x}^{T}(T) \mathbf{G} \mathbf{x}(T)+2 \grave{\eta} \mathbf{x}(T)\right]\right\},
$$

where $\mathbf{Q}=\operatorname{diag}(Q, \ldots, Q)-\frac{1}{N} \mathbf{1 1}^{T} \otimes Q_{\Gamma}, \mathbf{G}=\operatorname{diag}(G, \ldots, G)-\frac{1}{N} \mathbf{1 1}^{T} \otimes G_{\hat{\Gamma}}, \check{\eta}=\mathbf{1} \otimes \eta_{\Gamma}$ and $\grave{\eta}=\mathbf{1} \otimes \hat{\eta}_{\hat{\Gamma}}, Q_{\Gamma} \triangleq$ $\Gamma^{T} Q+Q \Gamma-\Gamma^{T} Q \Gamma, G_{\hat{\Gamma}} \triangleq \hat{\Gamma}^{T} G+G \hat{\Gamma}-\hat{\Gamma}^{T} G \hat{\Gamma}, \eta_{\Gamma}=Q \eta-\Gamma^{T} Q \eta$ and $\hat{\eta}_{\hat{\Gamma}}=G \hat{\eta}-\hat{\Gamma}^{T} G \hat{\eta}$.

For our further analysis, we have the following assumption:

(A5) The map $f \mapsto \check{\mathcal{J}}_{\text {soc }}^{F}(f)$ is uniformly convex.

Next, we give a necessary and sufficient condition which is useful in future discussion.

Proposition 3.1. The following statements are equivalent: (i) (A5) holds true. (ii) The following equation

$$
\dot{\boldsymbol{P}}+\check{\boldsymbol{A}}^{T} \boldsymbol{P}+\boldsymbol{P} \check{\boldsymbol{A}}-\boldsymbol{P}(\mathbf{1} \otimes I)\left(N R_{2}\right)^{-1}\left(\mathbf{1}^{T} \otimes I\right) \boldsymbol{P}-\boldsymbol{Q}=\boldsymbol{0}, \quad \boldsymbol{P}(T)=-\boldsymbol{G},
$$

admits a solution in $C^{1}\left([0, T] ; \mathbb{S}^{n N}\right)$. (iii) The equation

$$
\dot{P}+P(A+F)+(A+F)^{T} P-P R_{2}^{-1} P-\left(Q-Q_{\Gamma}\right)=0, \quad P(T)=-\left(G-G_{\hat{\Gamma}}\right),
$$

admits a solution in $C^{1}\left([0, T] ; \mathbb{S}^{n}\right)$.

(iv) $\operatorname{det}\left\{(0, I) e^{\mathbb{A} t}\left(\begin{array}{l}0 \\ I\end{array}\right)\right\}>0, \forall t \in[0, T]$, holds, where

$$
\mathbb{A}=\left(\begin{array}{cc}
A+F+R_{2}^{-1} G & -R_{2}^{-1} \\
\breve{Q} & -\left(A+F+R_{2}^{-1} G\right)^{T}
\end{array}\right)
$$

and $\breve{Q}=G R_{2}^{-1} G+(I-\Gamma)^{T} Q(I-\Gamma)+(A+F)^{T} G+G(A+F)$.

Proof. (i) $\Longleftrightarrow$ (ii) is proved in Theorem 4.5 of [40]. By Theorem 4.5 of [40], we obtain (i) $\Longleftrightarrow$ (iii). Moreover, we construct an auxiliary control problem

$$
\left\{\begin{array}{l}
\mathrm{d} y=((A+F) y+g) \mathrm{d} t, \quad y(0)=0, \\
\check{\mathcal{J}}_{\text {soc }}^{\prime} F(g)=\sum_{i=1}^{N} \mathbb{E}\left\{\int_{0}^{T}\left(-y^{T} \hat{Q} y+g^{T} R_{2} g\right) \mathrm{d} t-\left[y^{T}(T) \hat{G} y(T)\right]\right\},
\end{array}\right.
$$

where $\hat{Q}=(I-\Gamma)^{T} Q(I-\Gamma)$ and $\hat{G}=(I-\hat{\Gamma})^{T} G(I-\hat{\Gamma})$. Let $p$ be the adjoint equation of state $y$ and

$$
\mathrm{d} p=-\left[(A+F)^{T} p-\hat{Q} y\right] \mathrm{d} t, \quad p(T)=-\hat{G} y .
$$

By Itô formula to $\langle p, y\rangle$, we have

$$
\sum_{i=1}^{N} \mathbb{E}(\langle p(T), y(T)\rangle-\mathbb{E}\langle p(0), y(0)\rangle)=\sum_{i=1}^{N} \mathbb{E} \int_{0}^{T}\langle-\hat{Q} y, y\rangle+\langle p, g\rangle \mathrm{d} t .
$$


Thus, $\check{\mathcal{J}}_{\text {soc }}^{\prime} F(g)=0$ is equivalent to $g=-R_{2}^{-1} p$. Considering system

$$
\begin{cases}\mathrm{d} y=\left[(A+F) y-R_{2}^{-1} p\right] \mathrm{d} t, & y(0)=0 \\ \mathrm{~d} p=-\left[(A+F)^{T} p-\hat{Q} y\right] \mathrm{d} t, & p(T)=-\hat{G} y\end{cases}
$$

and letting $p=P y+\kappa$, one can obtain that

$$
\mathrm{d} p=\dot{P} y d t+P\left((A+F) y-R_{2}^{-1} p\right) \mathrm{d} t+d \kappa=-\left[(A+F)^{T} p-\hat{Q} y\right] \mathrm{d} t .
$$

Hence, $P$ and $\kappa$ should be the solution to

$$
\left\{\begin{array}{l}
\dot{P}+P(A+F)+(A+F)^{T} P-P R_{2}^{-1} P-\hat{Q}=0, \quad P(T)=-\hat{G}, \\
\dot{\kappa}+\left[(A+F)^{T}-P R_{2}^{-1}\right] \kappa=0, \quad \kappa(T)=0 .
\end{array}\right.
$$

For (iv) $\Longrightarrow$ (iii) is proved in Theorem 4.3 of [37]. On the other hand, we suppose (iii) holds. By Proposition 5.5 and Theorem 6.1 of Chapter 6 on [50], linear forward-backward ordinary differential equation (3.4) is solvable. Set $\tilde{p}=p+\hat{G} y$, then (3.4) can be rewritten as

$$
\left\{\begin{array}{l}
\mathrm{d} y=\left[\left(A+F+R_{2}^{-1} \hat{G}\right) y-R_{2}^{-1} \tilde{p}\right] \mathrm{d} t, \quad y(0)=0, \\
\mathrm{~d} \tilde{p}=\left[\left(\hat{Q}+(A+F)^{T} \hat{G}+\hat{G}(A+F)+G R_{2}^{-1} G\right) y-\left(A+F+R_{2}^{-1} \hat{G}\right)^{T} \tilde{p}\right] \mathrm{d} t, \quad \tilde{p}(T)=0,
\end{array}\right.
$$

which implies

$$
\left\{\begin{array}{l}
\mathrm{d}\left(\begin{array}{c}
y \\
\tilde{p}
\end{array}\right)=\left\{\mathbb{A}\left(\begin{array}{c}
y \\
\tilde{p}
\end{array}\right)+\mathbb{C} \beta\right\} \mathrm{d} t+\left\{\mathbb{A}_{1}\left(\begin{array}{c}
y \\
\tilde{p}
\end{array}\right)+\mathbb{C}_{1} \beta\right\} \mathrm{d} W(t), \\
y(0)=0, \quad \tilde{p}(T)=0
\end{array}\right.
$$

where $\mathbb{A}$ satisfies (3.3),

$$
\mathbb{C}=\left(\begin{array}{l}
0 \\
0
\end{array}\right), \quad \mathbb{A}_{1}=\left(\begin{array}{ll}
0 & 0 \\
0 & 0
\end{array}\right), \quad \mathbb{C}_{1}=\left(\begin{array}{l}
0 \\
0
\end{array}\right)
$$

By Theorem 3.7 of [37], we can obtain that (iii) $\Longrightarrow$ (iv). Then, the proposition follows.

Example 3.2. Consider Proposition 3.1 with parameters $A=B=R_{2}=1, F=-2, \Gamma=0.5, Q=4, G=0$, $T=1$. Then, by (3.2) we have

$$
P(t)=-\frac{1}{t-2}-1, \quad t \in[0,1] .
$$

$P(t)$ is well defined on $[0,1]$. And by the local Lipschitz continuity property of (3.2), (3.5) is unique. Furthermore, one can obtain that

$$
\mathbb{A}=\left(\begin{array}{cc}
-1 & -1 \\
1 & 1
\end{array}\right), \quad e^{\mathbb{A} t}=\left(\begin{array}{cc}
1-t & -t \\
t & t+1
\end{array}\right)
$$

and

$$
\operatorname{det}\left\{(0,1) e^{\mathbb{A} t}\left(\begin{array}{l}
0 \\
1
\end{array}\right)\right\}=t+1>0, \quad \forall t \in[0,1]
$$


which implies $(i i i) \Longleftrightarrow(i v)$.

According to above discussion, we have following theorem.

Theorem 3.3. Suppose that (A1)-(A4) hold. Then (P2a) has a unique minimizer if and only if (A5) hold and the following FBSDE admits a unique solution,

$$
\left\{\begin{array}{l}
\mathrm{d} \check{x}_{i}=\left(A \check{x}_{i}+B \check{u}_{i}+F \check{x}^{(N)}+\check{f}\right) \mathrm{d} t+\sigma \mathrm{d} W_{i}, \\
\mathrm{~d} \check{p}_{i}=-\left[A^{T} \check{p}_{i}+F^{T} \check{p}^{(N)}-\left(Q-Q_{\Gamma}\right) \check{x}^{(N)}+\eta_{\Gamma}\right] \mathrm{d} t+\sum_{j=1}^{N} \beta_{i}^{j} \mathrm{~d} W_{j}, \\
\check{x}_{i}(0)=\xi_{i}, \quad \check{p}_{i}(T)=-G \check{x}_{i}(T)+G_{\hat{\Gamma}} \check{x}^{(N)}(T)+\hat{\eta}_{\hat{\Gamma}}
\end{array}\right.
$$

where $p^{(N)}(t)=\frac{1}{N} \sum_{i=1}^{N} p_{i}(t)$ and $\check{f}=-R_{2}^{-1} p^{(N)}$.

Proof. By the similar argument in Theorem 3.1 of [48], we could obtain the result.

By taking average of (3.6) and letting $u^{(N)}=\frac{1}{N} \sum_{i=1}^{N} u_{i}$, we have following equations:

$$
\left\{\begin{array}{l}
\mathrm{d} \check{x}^{(N)}=\left((A+F) \check{x}^{(N)}+B \check{u}^{(N)}-R_{2}^{-1} \check{p}^{(N)}\right) \mathrm{d} t+\frac{1}{N} \sum_{i=1}^{N} \sigma \mathrm{d} W_{i}, \quad \check{x}^{(N)}(0)=\frac{1}{N} \sum_{i=1}^{N} \xi_{i}, \\
\mathrm{~d} \check{p}^{(N)}=-\left[(A+F)^{T} \check{p}^{(N)}-\left(Q-Q_{\Gamma}\right) \check{x}^{(N)}+\eta_{\Gamma}\right] \mathrm{d} t+\frac{1}{N} \sum_{i=1}^{N} \sum_{j=1}^{N} \beta_{i}^{j} \mathrm{~d} W_{j}, \\
\check{p}^{(N)}(T)=-\left(G-G_{\hat{\Gamma}}\right) \check{x}^{(N)}(T)+\hat{\eta}_{\hat{\Gamma}} .
\end{array}\right.
$$

Now we discuss the feedback form of disturbance in (P2a). We make the ansatz $\check{p}^{(N)}=\bar{P} \check{x}^{(N)}+\check{s}$, where $\bar{P} \in C^{1}\left([0, T] ; \mathbb{S}^{n}\right)$ is a matrix-value function and $\check{s} \in C^{1}\left([0, T] ; \mathbb{R}^{n}\right)$. Combining this equation and (3.7), one can obtain that

$$
\begin{aligned}
\mathrm{d} \check{p}^{(N)} & =\dot{\bar{P}} \check{x}^{(N)} \mathrm{d} t+\bar{P}\left(\left(A+F-R_{2}^{-1} P\right) \check{x}^{(N)}+B \check{u}^{(N)}-R_{2}^{-1} \check{s}\right) \mathrm{d} t+\frac{\bar{P}}{N} \sum_{i=1}^{N} \sigma \mathrm{d} W_{i}+\mathrm{d} \check{s} \\
& =-\left[(A+F)^{T}\left(\bar{P} \check{x}^{(N)}+\check{s}\right)-\left(Q-Q_{\Gamma}\right) \check{x}^{(N)}+\eta_{\Gamma}\right] \mathrm{d} t+\frac{1}{N} \sum_{i=1}^{N} \sum_{j=1}^{N} \beta_{i}^{j} \mathrm{~d} W_{j} .
\end{aligned}
$$

Hence, $\bar{P}(\cdot)$ is a solution of

$$
\dot{\bar{P}}+\bar{P}(A+F)+(A+F)^{T} \bar{P}-\bar{P} R_{2}^{-1} \bar{P}-\left(Q-Q_{\Gamma}\right)=0, \quad \bar{P}(T)=-\left(G-G_{\hat{\Gamma}}\right) .
$$

and $\check{s}(\cdot)$ is the solution of the following BSDE:

$$
\mathrm{d} \check{s}+\left[(A+\bar{F})^{T} \check{s}+\bar{P} B \check{u}^{(N)}+\eta_{\Gamma}\right] \mathrm{d} t+\frac{1}{N} \sum_{j=1}^{N} \sum_{i=1}^{N}\left(\frac{\bar{P} \sigma}{N}-\beta_{i}^{j}\right) \mathrm{d} W_{j}=0, \quad \check{s}(T)=\hat{\eta}_{\hat{\Gamma}},
$$

where $\bar{F}=F-R_{2}^{-1} \bar{P}$. Thus, $\check{f}=-R_{2}^{-1}\left(\bar{P} \check{x}^{(N)}+\check{s}\right)$. We can easily see that $P$ in (3.2) is equal to $\bar{P}$ here. In what follows, $\bar{P}$ will be substituted by $P$. 


\section{Distributed STRATEGy DESIGN}

After applying the worst disturbance $\check{f}$, one can obtain the following optimal control problem. (P3): Minimize $\mathcal{J}_{\text {soc }}^{F}(u, \check{f}(u))$ over $\left\{u=\left(u_{1}, \ldots, u_{N}\right) \in \mathcal{U}_{c}^{F}\right\}$, where

$$
\left\{\begin{array}{l}
\mathrm{d} x_{i}=\left[A x_{i}+B u_{i}+\bar{F} x^{(N)}-R_{2}^{-1} s\right] \mathrm{d} t+\sigma \mathrm{d} W_{i}, \quad x_{i}(0)=\xi_{i}, \\
\mathrm{~d} s=-\left[(A+\bar{F})^{T} s+P B u^{(N)}+\eta_{\Gamma}\right] \mathrm{d} t+\frac{1}{N} \sum_{j=1}^{N} \sum_{i=1}^{N}\left(\beta_{i}^{j}-\frac{P \sigma}{N}\right) \mathrm{d} W_{j}, \quad s(T)=\hat{\eta}_{\hat{\Gamma}}
\end{array}\right.
$$

and

$$
\begin{aligned}
\mathcal{J}_{\text {soc }}^{F}(u)= & \frac{1}{2} \sum_{i=1}^{N} \mathbb{E} \int_{0}^{T}\left\{\left|x_{i}-\Gamma x^{(N)}-\eta\right|_{Q(t)}^{2}+\left|u_{i}\right|_{R_{1}}^{2}-\left|P(t) x^{(N)}+s\right|_{R_{2}^{-1}}^{2}\right\} \mathrm{d} t \\
& +\frac{1}{2} \sum_{i=1}^{N} \mathbb{E}\left|x_{i}(T)-\hat{\Gamma} x^{(N)}(T)-\hat{\eta}\right|_{G}^{2} .
\end{aligned}
$$

To solve (P3), we first give out a proposition.

Proposition 4.1. Suppose that (A1)-(A5) hold. If (P3) is uniformly convex in $u$, then (P3) has a set of optimal controls and the following FBSDE admits a set of solutions

$$
\left\{\begin{array}{l}
\mathrm{d} x_{i}=\left[A x_{i}+B u_{i}+\bar{F} x^{(N)}-R_{2}^{-1} s\right] \mathrm{d} t+\sigma \mathrm{d} W_{i}, \quad x_{i}(0)=\xi_{i}, \\
\mathrm{~d} s=-\left[(A+\bar{F})^{T} s+P B u^{(N)}+\eta_{\Gamma}\right] \mathrm{d} t+\frac{1}{N} \sum_{j=1}^{N} \sum_{i=1}^{N}\left(\beta_{i}^{j}-\frac{P \sigma}{N}\right) \mathrm{d} W_{j}, \quad s(T)=\hat{\eta}_{\hat{\Gamma}}, \\
\mathrm{d} k_{i}=\left[-A^{T} k_{i}-\bar{F}^{T} k^{(N)}-Q x_{i}+Q_{\Gamma} x^{(N)}+\eta_{\Gamma}+P R_{2}^{-1}\left(P x^{(N)}+s\right)\right] \mathrm{d} t+\zeta_{i}^{i} \mathrm{~d} W_{i}+\sum_{j \neq i} \zeta_{i}^{j} \mathrm{~d} W_{j}, \\
\mathrm{~d} l=\left[(A+\bar{F}) l+R_{2}^{-1}\left(k^{(N)}+P x^{(N)}+s\right)\right] \mathrm{d} t, \quad l(0)=0, \quad k_{i}(T)=G \bar{x}_{i}(T)-G_{\hat{\Gamma}} \bar{x}^{(N)}(T)-\hat{\eta}_{\hat{\Gamma}},
\end{array}\right.
$$

where $k^{(N)}=\frac{1}{N} \sum_{i=1}^{N} k_{i}$ and $R_{1} u_{i}+B^{T} k_{i}-B^{T} P l=0, i=1,2, \ldots, N$.

Proof. Let $u^{*}=\left\{u_{1}^{*}, u_{2}^{*}, \ldots, u_{N}^{*}\right\} \in \mathcal{U}_{c}^{F}$ be the unique centralized optimal control of the $N$ agents and $x^{*}=$ $\left\{x_{1}^{*}, x_{2}^{*}, \ldots, x_{N}^{*}\right\}$ be their unique corresponding states. We perturb $u^{*}$ and denote $\delta u=u-u^{*}, \delta u^{(N)}=u^{(N)}-$ $\left(u^{*}\right)^{(N)}, \delta x_{i}=x_{i}-x_{i}^{*}, \delta x^{(N)}=\frac{1}{N} \sum_{i=1}^{N} \delta x_{i}=\frac{1}{N} \sum_{i=1}^{N}\left(x_{i}-x_{i}^{*}\right)=\frac{1}{N} \sum_{i=1}^{N} x_{i}-\frac{1}{N} \sum_{i=1}^{N} x_{i}^{*}=x^{(N)}-\left(x^{*}\right)^{(N)}$, $\delta s=s-s^{*}$. Then we have

$$
\left\{\begin{array}{l}
\mathrm{d} \delta x_{i}=\left[A \delta x_{i}+B \delta u_{i}+\bar{F} \delta x^{(N)}-R_{2}^{-1} \delta s\right] \mathrm{d} t, \quad \delta x_{i}(0)=0 \\
\mathrm{~d} \delta s=-\left[(A+\bar{F})^{T} \delta s+P B \delta u^{(N)}\right] \mathrm{d} t+\frac{1}{N} \sum_{j=1}^{N} \sum_{i=1}^{N} \delta \beta_{i}^{j} \mathrm{~d} W_{j}, \quad \delta s(T)=0 .
\end{array}\right.
$$

By the same argument in Section 4.2, the Fréchet differential of the corresponding social cost functional is

$$
\delta \mathcal{J}_{\text {soc }}^{F}(\delta u)=\mathcal{J}_{\text {soc }}^{F}(u)-\mathcal{J}_{\text {soc }}^{F}\left(u^{*}\right)+o\left(\|\delta u\|_{L^{2}}\right)=\Lambda_{1}+\frac{1}{2} \Lambda_{2},
$$


where

$$
\begin{aligned}
\Lambda_{1} \triangleq & \sum_{i=1}^{N} \mathbb{E} \int_{0}^{T}\left\langle Q\left(x_{i}^{*}-\Gamma\left(x^{*}\right)^{(N)}-\eta\right), \delta x_{i}-\Gamma \delta x^{(N)}\right\rangle+\left\langle R_{1} u_{i}^{*}, \delta u_{i}\right\rangle-\left\langle R_{2}^{-1}\left(P\left(x^{*}\right)^{(N)}+\bar{s}\right),\right. \\
& \left.\left(P \delta x^{(N)}+\delta s\right)\right\rangle \mathrm{d} t+\sum_{i=1}^{N} \mathbb{E}\left\langle G\left(x_{i}^{*}(T)-\hat{\Gamma}\left(x^{*}\right)^{(N)}(T)-\hat{\eta}\right), \delta x_{i}(T)-\hat{\Gamma} \delta x^{(N)}(T)\right\rangle, \\
\Lambda_{2} \triangleq & \sum_{i=1}^{N} \mathbb{E} \int_{0}^{T}\left\{\left|\delta x_{i}-\Gamma \delta x^{(N)}\right|_{Q}^{2}+\left|\delta u_{i}\right|_{R_{1}}^{2}-\left|P \delta x^{(N)}+\delta s\right|_{R_{2}^{-1}}^{2}\right\} \mathrm{d} t+\sum_{i=1}^{N} \mathbb{E}\left|\delta x_{i}(T)-\hat{\Gamma} \delta x^{(N)}(T)\right|_{G}^{2} .
\end{aligned}
$$

Note that

$$
\begin{aligned}
& \sum_{i=1}^{N} \mathbb{E} \int_{0}^{T}\left\langle-Q\left(x_{i}^{*}-\Gamma\left(x^{*}\right)^{(N)}-\eta\right), \delta \Gamma x^{(N)}\right\rangle \mathrm{d} t=\mathbb{E} \int_{0}^{T}\left\langle\Gamma^{T} Q \sum_{i=1}^{N}\left(x_{i}^{*}-\Gamma\left(x^{*}\right)^{(N)}-\eta\right), \frac{1}{N} \sum_{j=1}^{N} \delta x_{j}\right\rangle \mathrm{d} t \\
= & \mathbb{E} \int_{0}^{T} \sum_{j=1}^{N}\left\langle\frac{\Gamma^{T} Q}{N} \sum_{i=1}^{N}\left(x_{i}^{*}-\Gamma\left(x^{*}\right)^{(N)}-\eta\right), \delta x_{j}\right\rangle \mathrm{d} t=\sum_{i=1}^{N} \mathbb{E} \int_{0}^{T}\left\langle\Gamma^{T} Q\left((I-\Gamma)\left(x^{*}\right)^{(N)}-\eta\right), \delta x_{i}\right\rangle \mathrm{d} t .
\end{aligned}
$$

and

$$
\begin{aligned}
& \sum_{i=1}^{N} \mathbb{E} \int_{0}^{T}-\left\langle R_{2}^{-1}\left(P\left(x^{*}\right)^{(N)}+\bar{s}\right),\left(P \delta x^{(N)}+\delta s\right)\right\rangle \mathrm{d} t \\
= & \sum_{i=1}^{N} \mathbb{E} \int_{0}^{T}-\left\langle P^{T} R_{2}^{-1}\left(P\left(x^{*}\right)^{(N)}+\bar{s}\right), \delta x_{i}\right\rangle-\left\langle R_{2}^{-1}\left(P\left(x^{*}\right)^{(N)}+\bar{s}\right), \delta s\right\rangle \mathrm{d} t .
\end{aligned}
$$

Let

$$
\left\{\begin{array}{l}
\mathrm{d} k_{i}=\alpha_{i} \mathrm{~d} t+\zeta_{i}^{i} \mathrm{~d} W_{i}+\sum_{j \neq i} \zeta_{i}^{j} \mathrm{~d} W_{j}, \quad k_{i}(T)=G x_{i}^{*}(T)-G_{\hat{\Gamma}}\left(x^{*}\right)^{(N)}(T)-\hat{\eta}_{\hat{\Gamma}} \\
\mathrm{d} l=\gamma \mathrm{d} t,+\nu_{i} \mathrm{~d} W_{i}+\sum_{j \neq i} \nu_{j} \mathrm{~d} W_{j}, \quad l(0)=0 .
\end{array}\right.
$$

where

$$
\left\{\begin{array}{l}
\alpha_{i}=-\left[A^{T} k_{i}+\bar{F}^{T} k^{(N)}+Q x_{i}^{*}-Q_{\Gamma}\left(x^{*}\right)^{(N)}-\eta_{\Gamma}-P R_{2}^{-1}\left(P\left(x^{*}\right)^{(N)}+\bar{s}\right)\right], \\
\gamma=(A+\bar{F}) l+R_{2}^{-1}\left(k^{(N)}+P\left(x^{*}\right)^{(N)}+\bar{s}\right), \quad \sum_{j=1}^{N} \nu_{j}=0
\end{array}\right.
$$

By Itô formula,

$$
\begin{aligned}
& \sum_{i=1}^{N} \mathbb{E}\left\langle k_{i}(T), \delta x_{i}(T)\right\rangle-\sum_{i=1}^{N} \mathbb{E}\left\langle k_{i}(0), \delta x_{i}(0)\right\rangle \\
= & \sum_{i=1}^{N} \mathbb{E} \int_{0}^{T}\left\langle\alpha_{i}+A^{T} k_{i}+\bar{F}^{T} k^{(N)}, \delta x_{i}\right\rangle+\left\langle B^{T} k_{i}, \delta u_{i}\right\rangle-\left\langle R_{2}^{-1} k^{(N)}, \delta s\right\rangle \mathrm{d} t,
\end{aligned}
$$


and

$$
\begin{aligned}
& \sum_{i=1}^{N} \mathbb{E}\langle l(T), \delta s(T)\rangle-\mathbb{E}\langle l(0), \delta s(0)\rangle=0 \\
= & \sum_{i=1}^{N} \mathbb{E} \int_{0}^{T}\langle\gamma-(A+\bar{F}) l, \delta s\rangle-\left\langle B^{T} P l, \delta u_{i}\right\rangle+\frac{1}{N}\left\langle\sum_{j=1}^{N} \nu_{j}, \sum_{i=1}^{N} \delta \beta_{i}^{j}\right\rangle \mathrm{d} t .
\end{aligned}
$$

Consequently by (4.4), (4.5), (4.7), (4.8) and (4.9), we have

$$
\Lambda_{1}=\sum_{i=1}^{N} \mathbb{E} \int_{0}^{T}\left\langle R_{1} u_{i}^{*}+B^{T} k_{i}-B^{T} P l, \delta u_{i}\right\rangle \mathrm{d} t .
$$

Thus, $\Lambda_{1}=0$ is equivalent to $R_{1} u_{i}^{*}+B^{T} k_{i}-B^{T} P l=0, i=1,2, \ldots, N$. Then, considering (4.1), (4.6) and (4.7), we have (4.3). The proposition follows.

It follows from (4.3) that

$$
\left\{\begin{array}{l}
\mathrm{d} x^{(N)}=\left[(A+\bar{F}) x^{(N)}+B u^{(N)}-R_{2}^{-1} s\right] \mathrm{d} t+\frac{1}{N} \sum_{i=1}^{N} \sigma \mathrm{d} W_{i}, \quad x^{(N)}(0)=\frac{1}{N} \sum_{i=1}^{N} \xi_{i}, \\
\mathrm{~d} k^{(N)}=\left[-(A+\bar{F})^{T} k^{(N)}-\left(Q-Q_{\Gamma}\right) \bar{x}^{(N)}+\eta_{\Gamma}+P R_{2}^{-1}\left(P x^{(N)}+s\right)\right] \mathrm{d} t+\frac{1}{N} \sum_{i=1}^{N} \sum_{j=1}^{N} \zeta_{i}^{j} \mathrm{~d} W_{j}, \\
k^{(N)}(T)=\left(G-G_{\hat{\Gamma}}\right) x^{(N)}(T)-\hat{\eta}_{\hat{\Gamma}} .
\end{array}\right.
$$

To discuss the state feedback form of the optimal controls we solved in (P3), we consider the following nonhomogeneous relationships:

$$
k_{i}=K x_{i}+L x^{(N)}+M l+\varphi, \quad k^{(N)}=(K+L) x^{(N)}+M l+\varphi, \quad s=\bar{M} l+\bar{L} x^{(N)}+\phi,
$$

where $K, L, M, \bar{M}, \bar{L} \in C^{1}\left([0, T] ; \mathbb{R}^{n \times n}\right)$ and $\varphi, \phi \in C^{1}\left([0, T] ; \mathbb{R}^{n}\right)$. By (4.11), (4.3) and (4.10), we have

$$
\begin{aligned}
\mathrm{d} k_{i}= & \dot{K} x_{i} \mathrm{~d} t+K\left[A x_{i}+B u_{i}+\bar{F} x^{(N)}-R_{2}^{-1} s\right] \mathrm{d} t+K \sigma \mathrm{d} W_{i}+\dot{L} x^{(N)} \mathrm{d} t+L\left[(A+\bar{F}) x^{(N)}+B u^{(N)}\right. \\
& \left.-R_{2}^{-1} s\right] \mathrm{d} t+\frac{L}{N} \sum_{i=1}^{N} \sigma \mathrm{d} W_{i}+\dot{M} l \mathrm{~d} t+M\left((A+\bar{F}) l+R_{2}^{-1}\left(k^{(N)}+P x^{(N)}+s\right)\right) \mathrm{d} t+\mathrm{d} \varphi \\
= & -\left[A^{T} k_{i}+\bar{F}^{T} k^{(N)}+Q\left(x_{i}-\Gamma x^{(N)}-\eta\right)-\Gamma^{T} Q\left((I-\Gamma) x^{(N)}-\eta\right)\right. \\
& \left.-P R_{2}^{-1}\left(P x^{(N)}+s\right)\right] \mathrm{d} t+\sum_{j=1}^{N} \zeta_{i}^{j} \mathrm{~d} W_{j}, \\
\mathrm{~d} s= & \dot{\bar{M}} l \mathrm{~d} t+\bar{M}\left[(A+\bar{F}) l+R_{2}^{-1}\left(k^{(N)}+P x^{(N)}+s\right)\right] \mathrm{d} t+\dot{\bar{L}} x^{(N)} \mathrm{d} t+\bar{L}\left((A+\bar{F}) x^{(N)}+B u^{(N)}\right. \\
& \left.-R_{2}^{-1} s\right) \mathrm{d} t+\frac{\bar{L}}{N} \sum_{i=1}^{N} \sigma \mathrm{d} W_{i}+\mathrm{d} \phi \\
= & -\left[(A+\bar{F})^{T}\left(\bar{M} l+\bar{L} x^{(N)}+\phi\right)+P B u^{(N)}+(I-\Gamma)^{T} Q \eta\right] \mathrm{d} t+\frac{1}{N} \sum_{j=1}^{N} \sum_{i=1}^{N}\left(\beta_{i}^{j}-\frac{P \sigma}{N}\right) \mathrm{d} W_{j} .
\end{aligned}
$$


Comparing the diffusion terms in (4.12), we have the following results: $\left(K+\frac{L}{N}\right) \sigma=\zeta_{i}^{i}, \frac{L}{N} \sigma=\zeta_{i}^{j}, j \neq i$, $\frac{\bar{L}}{N} \sum_{i=1}^{N} \sigma=\frac{1}{N} \sum_{j=1}^{N} \sum_{i=1}^{N}\left(\beta_{i}^{j}-\frac{P \sigma}{N}\right)$. Hence, combining (4.11) and the equation $R_{1} \bar{u}_{i}+B^{T} k_{i}-B^{T} P l=0$, we have

$$
\left\{\begin{array}{l}
u_{i}=-R_{1}^{-1}\left\{B^{T} K x_{i}+B^{T} L x^{(N)}+B^{T} \varphi-B^{T}(P-M) l\right\} \\
u^{(N)}=-R_{1}^{-1}\left\{B^{T}(K+L) x^{(N)}+B^{T} \varphi-B^{T}(P-M) l\right\} .
\end{array}\right.
$$

Thus, using the same argument from (3.8) to (3.10), it follows that $K(\cdot)$ is a solution of

$$
\dot{K}+K A+A^{T} K-\left(B^{T} K\right)^{T} R_{1}^{-1} B^{T} K+Q=0, \quad K(T)=G,
$$

and $L(\cdot), M(\cdot), \bar{L}(\cdot), \bar{M}(\cdot)$ satisfy

$$
\left\{\begin{array}{l}
\dot{L}+L(A+\bar{F})+(A+\bar{F})^{T} L+K \bar{F}+\bar{F}^{T} K-P R_{2}^{-1}(P+\bar{L})+M R_{2}^{-1}(K+L+P+\bar{L}) \\
\quad-(K+L) R_{2}^{-1} \bar{L}-\left(B^{T}(K+L)\right)^{T} R_{1}^{-1} B^{T}(K+L)+\left(B^{T} K\right)^{T} R_{1}^{-1} B^{T} K-Q_{\Gamma}=0, \quad L(T)=-G_{\hat{\Gamma}} \\
\dot{\bar{L}}+\bar{L}(A+\bar{F})+(A+\bar{F})^{T} \bar{L}-\bar{L} R_{2}^{-1} \bar{L}+\bar{M} R_{2}^{-1}(K+L+P+\bar{L}) \\
\quad-\left(B^{T}(P+\bar{L})\right)^{T} R_{1}^{-1} B^{T}(K+L)=0, \quad \bar{L}(T)=0 \\
\dot{M}+M(A+\bar{F})+(A+\bar{F})^{T} M-(K+L+P) R_{2}^{-1} \bar{M}+M R_{2}^{-1}(M+\bar{M}) \\
\quad+\left(B^{T}(K+L)\right)^{T} R_{1}^{-1} B^{T}(P-M)=0, \quad M(T)=0 \\
\dot{\bar{M}}+\bar{M}(A+\bar{F})+(A+\bar{F})^{T} \bar{M}+\bar{M} R_{2}^{-1}(M+\bar{M})-\bar{L} R_{2}^{-1} \bar{M} \\
\quad+\left(B^{T}(P+\bar{L})\right)^{T} R_{1}^{-1} B^{T}(P-M)=0, \quad \bar{M}(T)=0,
\end{array}\right.
$$

and $\varphi(\cdot), \phi(\cdot)$ satisfy

$$
\left\{\begin{array}{l}
\mathrm{d} \varphi+\left(M R_{2}^{-1}+A+\bar{F}\right)^{T} \varphi-\left(B^{T}(K+L)\right)^{T} R_{1}^{-1} B^{T} \varphi-(K+L+P-M) R_{2}^{-1} \phi-\eta_{\Gamma}=0, \quad \varphi(T)=-\hat{\eta}_{\hat{\Gamma}} \\
\mathrm{d} \phi+\left((\bar{M}-\bar{L}) R_{2}^{-1}+A+\bar{F}\right)^{T} \phi-P B R_{2}^{-1} B^{T} \varphi+\bar{M} R_{2}^{-1} \varphi+\eta_{\Gamma}=0, \quad \phi(T)=\hat{\eta}_{\hat{\Gamma}}
\end{array}\right.
$$

Remark 4.2. Equation (4.15) are non-symmetric Riccati equations or Riccati-like equations. For more details about their property and solvabibility, readers could refer to [20, 30, 32].

If $L, M, \bar{M} \in C^{1}\left([0, T] ; \mathbb{S}^{n}\right)$ are the unique solutions to (4.15), we have following result:

Proposition 4.3. Suppose that (A1)-(A5) hold. If $L, M, \bar{M} \in C^{1}\left([0, T] ; \mathbb{S}^{n}\right)$, then $\bar{L}=-M$ and the original four coupled Riccati-like equations can be simplified to three coupled equations.

Proof. According to equation (4.14), $K$ is symmetric. It follows from taking transpose on $M$ and multiply -1 on both sides in (4.15) that,

$$
\begin{gathered}
-\dot{M}^{T}+(A+\bar{F})^{T}(-M)^{T}+(-M)^{T}(A+\bar{F})+\bar{M}^{T} R_{2}^{-1}(K+L+P-M)^{T}-M^{T} R_{2}^{-1} M^{T} \\
-\left(B^{T}\left(P-M^{T}\right)\right)^{T} R_{1}^{-1} B^{T}(K+L)=0, \quad-M^{T}(T)=0,
\end{gathered}
$$


Since $L, M, \bar{M} \in C^{1}\left([0, T] ; \mathbb{S}^{n}\right)$, we have $\bar{L}=-M^{T}=-M$. Putting this result into system (4.15), it could be simplified as

$$
\left\{\begin{array}{l}
\dot{L}+L(A+\bar{F})+(A+\bar{F})^{T} L+K \bar{F}+\bar{F}^{T} K-P R_{2}^{-1} P-M R_{2}^{-1} M+M R_{2}^{-1}(K+L+P) \\
\quad+(K+L+P) R_{2}^{-1} M-\left(B^{T}(K+L)\right)^{T} R_{1}^{-1} B^{T}(K+L)+\left(B^{T} K\right)^{T} R_{1}^{-1} B^{T} K-Q_{\Gamma}=0 \\
\dot{M}+M(A+\bar{F})+(A+\bar{F})^{T} M-(K+L+P-M) R_{2}^{-1} \bar{M}+M R_{2}^{-1} M \\
\quad+\left(B^{T}(K+L)\right)^{T} R_{1}^{-1} B^{T}(P-M)=0, \quad M(T)=0 \\
\dot{\bar{M}}+\bar{M}(A+\bar{F})+(A+\bar{F})^{T} \bar{M}+\bar{M} R_{2}^{-1} M+M R_{2}^{-1} \bar{M}+\bar{M} R_{2}^{-1} \bar{M} \\
\quad+\left(B^{T}(P-M)\right)^{T} R_{1}^{-1} B^{T}(P-M)=0, \quad \bar{M}(T)=0, \quad L(T)=-G_{\hat{\Gamma}}
\end{array}\right.
$$

The proposition follows.

By a similar argument of Lemma 2.1 in [46], if (A1)-(A5) hold, there exists a constant $\delta>0$ such that $R_{1}(t)>\delta I$ and $R_{2}(t)>\delta I$. Then, for $Q \geq 0,(\mathbf{P 3})$ is uniformly convex. However, when $Q$ is indefinite, we have following result.

Lemma 4.4. (P3) has uniform convexity if equations (4.14)-(4.16) has a solution, respectively.

Proof. Similar to Proposition 3.1 in [48] and Section 3 in [26], we first let $\dot{u}_{i} \in \mathcal{U}_{c}^{F}, \dot{s} \in C^{1}\left([0, T] ; \mathbb{R}^{n}\right)$ and consider the system

$$
\left\{\begin{array}{l}
\mathrm{d} y_{i}=A y_{i}+B \dot{u}_{i}+F y^{(N)}-R_{2}^{-1}\left(P y^{(N)}+\dot{s}\right) \mathrm{d} t, \quad y_{i}(0)=0, \\
\mathrm{~d} y^{(N)}=A y^{(N)}+B \dot{u}^{(N)}+F y^{(N)}-R_{2}^{-1}\left(P y^{(N)}+\dot{s}\right) \mathrm{d} t, \quad y^{(N)}(0)=0 \\
\mathrm{~d}\left(y_{i}-y^{(N)}\right)=A\left(y_{i}-y^{(N)}\right)+B\left(\dot{u}_{i}-\dot{u}^{(N)}\right) \mathrm{d} t, \quad\left(y_{i}-y^{(N)}\right)(0)=0 .
\end{array}\right.
$$

By (3.9), (4.14) and using Itô formula to $\left|y_{i}-y^{(N)}\right|_{K}^{2}$ and $\left|y_{i}-y^{(N)}\right|_{P}^{2}$, we have

$$
\begin{aligned}
& \mathbb{E}\left|y_{i}(T)-y^{(N)}(T)\right|_{G}^{2}=\mathbb{E}\left|y_{i}(T)-y^{(N)}(T)\right|_{K(T)}^{2}-\mathbb{E}\left|y_{i}(0)-y^{(N)}(0)\right|_{K(0)}^{2} \\
= & \mathbb{E} \int_{0}^{T}\left\langle\left(\left(B^{T} K\right)^{T} R_{1}^{-1} B^{T} K-Q\right)\left(y_{i}-y^{(N)}\right), y_{i}-y^{(N)}\right\rangle+2\left\langle\dot{u}_{i}-\dot{u}^{(N)}, B^{T} K\left(y_{i}-y^{(N)}\right)\right\rangle,
\end{aligned}
$$

and

$$
\begin{aligned}
& -\mathbb{E}\left|y^{(N)}(T)\right|_{G-G_{\hat{\Gamma}}}^{2}=\mathbb{E}\left|y^{(N)}(T)\right|_{P(T)}^{2}-\mathbb{E}\left|y^{(N)}(0)\right|_{P(0)}^{2} \\
& =\mathbb{E} \int_{0}^{T}\left\langle\left(P R_{2}^{-1} P+Q-Q_{\Gamma}\right) y^{(N)}, y^{(N)}\right\rangle+2\left\langle\dot{u}^{(N)}, B^{T} P y^{(N)}\right\rangle-2\left\langle\dot{s}, R_{2}^{-1} P y^{(N)}\right\rangle .
\end{aligned}
$$


By Lemma 2.1 in [46], we know that $\sum_{i=1}^{N} \mathbb{E} \int_{0}^{T}\left|y_{i}\right|^{2} \mathrm{~d} t \leq \frac{c}{N} \sum_{i=1}^{N} \mathbb{E} \int_{0}^{T}\left|\dot{u}_{i}\right|^{2} \mathrm{~d} t, \quad \sum_{i=1}^{N} \mathbb{E} \int_{0}^{T}|\dot{s}|^{2} \mathrm{~d} t \leq$ $\frac{c}{N} \sum_{i=1}^{N} \mathbb{E} \int_{0}^{T}\left|\dot{u}_{i}\right|^{2} \mathrm{~d} t$. By Lemma 2.3 in [40] and Proposition 3.1,

$$
\begin{aligned}
& \sum_{i=1}^{N} \mathbb{E} \int_{0}^{T}\left(\left|y_{i}-\Gamma y^{(N)}\right|_{Q}^{2}+\left|\dot{u}_{i}\right|_{R_{1}}^{2}-\left|P y^{(N)}+\dot{s}\right|_{R_{2}^{-1}}^{2}\right) \mathrm{d} t+\sum_{i=1}^{N} \mathbb{E}\left|y_{i}(T)-\hat{\Gamma} y^{(N)}(T)\right|_{G}^{2} \\
& =\sum_{i=1}^{N} \mathbb{E} \int_{0}^{T}\left(\left|y_{i}-\Gamma y^{(N)}\right|_{Q}^{2}+\left|y^{(N)}\right|_{Q-Q_{\Gamma}}^{2}+\left|\dot{u}_{i}-\dot{u}^{(N)}\right|_{R_{1}}^{2}+\left|\dot{u}^{(N)}\right|_{R_{1}}^{2}-\left|P y^{(N)}\right|_{R_{2}^{-1}}^{2}\right. \\
& \left.-2\left\langle\dot{s}, R_{2}^{-1} P y^{(N)}\right\rangle-|\dot{s}|_{R_{2}^{-1}}^{2}\right) \mathrm{d} t+\sum_{i=1}^{N} \mathbb{E}\left|y_{i}(T)-y^{(N)}(T)\right|_{G}^{2}+\left|y^{(N)}(T)\right|_{G-G_{\hat{\Gamma}}}^{2} \\
& =\sum_{i=1}^{N} \mathbb{E} \int_{0}^{T}\left(\left|\dot{u}_{i}-\dot{u}^{(N)}+R_{1}^{-1} B^{T} K\left(y_{i}-y^{(N)}\right)\right|_{R_{1}}^{2}+\left|\dot{u}^{(N)}-R_{1}^{-1} B P y^{(N)}\right|_{R_{1}}^{2}\right. \\
& \left.-\left|P y^{(N)}\right|_{B^{T} R_{1}^{-1} B+2 R_{2}^{-1}-\mid s^{2}}^{2} R_{2}^{-1}\right) \mathrm{d} t \\
& \geq \sum_{i=1}^{N} \mathbb{E} \int_{0}^{T}\left(\left|\dot{u}_{i}+R_{1}^{-1} B^{T} K y_{i}-R_{1}^{-1} B(P+K) y^{(N)}\right|_{R_{1}}^{2}-\frac{c}{N}\left|\dot{u}_{i}\right|^{2}\right) \mathrm{d} t \geq \delta \sum_{i=1}^{N} \mathbb{E} \int_{0}^{T}\left|\dot{u}_{i}\right|^{2} \mathrm{~d} t .
\end{aligned}
$$

The lemma follows.

For further proofs, we have the following assumption:

(A6) Assume that (4.14)-(4.15) admit unique solutions.

Then, by above discussion, we have following theorem.

Theorem 4.5. Suppose that (A1)-(A6) hold. Then (P3) is uniquely solvable with the optimal control $u_{i}$ in (4.13).

Proof. Since (4.14)-(4.16) has a solution, respectively, the system (4.3) is decoupled and solvable (see the Theorem 3.7 and Theorem 4.3 of Chapter 2 in [37]). By Lemma 4.4, (P3) has uniform convexity and can achieves an optimal control, where $u_{i}=-R_{1}^{-1} B^{T}\left\{K x_{i}+L x^{(N)}+\varphi-(P-M) l\right\}$.

We use $\hat{x}, \hat{l}$ to approximate $x^{(N)}, l$ in $(4.3)$ and (4.10), respectively.

$$
\left\{\begin{array}{l}
\mathrm{d} \hat{x}=\left\{\left[A+\bar{F}-B R_{1}^{-1} B^{T}(K+L)-R_{2}^{-1} \bar{L}\right] \hat{x}-B R_{1}^{-1} B^{T} \varphi+\left[B R_{1}^{-1} B^{T}(P-M)-R_{2}^{-1} \bar{M}\right] \hat{l}-R_{2}^{-1} \phi\right\} \mathrm{d} t, \\
\mathrm{~d} \hat{l}=\left[\left(A+\bar{F}+R_{2}^{-1}(M+\bar{M})\right) \hat{l}+R_{2}^{-1}((K+L+P+\bar{L}) \hat{x}+\varphi+\phi)\right] \mathrm{d} t, \quad \hat{l}(0)=0, \quad \hat{x}(0)=\hat{\xi},
\end{array}\right.
$$

where $K, L, \bar{L}, M, \bar{M}, \varphi$ and $\phi$ are determined by (4.14)-(4.16). Then, according to Theorem 4.5, one can obtain the decentralized control law for the $i$ th agent

$$
\bar{u}_{i}=-R_{1}^{-1} B^{T}\left[K \bar{x}_{i}+L \hat{x}+\varphi-(P-M) \hat{l}\right]
$$


Meanwhile, we have the decentralized terms $\bar{k}_{i}=K \bar{x}_{i}+L \hat{x}+M \hat{l}+\varphi, \bar{s}=\bar{M} \bar{l}+\bar{L} \bar{x}^{(N)}+\phi$, and $\hat{s}=\bar{M} \hat{l}+\bar{L} \hat{x}+\phi$. By applying (4.18), we have the following closed-loop system

$$
\left\{\begin{aligned}
\mathrm{d} \bar{x}_{i}= & \left\{\left[A-B R_{1}^{-1} B^{T} K\right] \bar{x}_{i}+\left(\bar{F}-R_{2}^{-1} \bar{L}\right) \bar{x}^{(N)}-B R_{1}^{-1} B^{T} L \hat{x}-B R_{1}^{-1} B^{T} \varphi+B R_{1}^{-1} B^{T}(P-M) \hat{l}\right. \\
& \left.-R_{2}^{-1}(\bar{M} \bar{l}+\phi)\right\} \mathrm{d} t+\sigma \mathrm{d} W_{i}, \quad \bar{x}_{i}(0)=\xi_{i} \\
\mathrm{~d} \bar{s}= & -\left\{(A+\bar{F})^{T} \bar{s}+\left(B^{T} P\right)^{T} R_{1}^{-1} B^{T} K \bar{x}^{(N)}+\left(B^{T} P\right)^{T} R_{1}^{-1} B^{T} L \hat{x}+\left(B^{T} P\right)^{T} R_{1}^{-1} B^{T} \varphi\right. \\
& \left.-\left(B^{T} P\right)^{T} R_{1}^{-1} B^{T}(P-M) \hat{l}-(I-\Gamma)^{T} Q \eta\right\} \mathrm{d} t+\frac{1}{N} \sum_{j=1}^{N} \sum_{i=1}^{N}\left(\frac{P \sigma}{N}-\beta_{i}^{j}\right) d W_{j}, \quad \bar{s}(T)=\hat{\eta}_{\hat{\Gamma}}, \\
\mathrm{d} \bar{l}= & {\left[\left(A+\bar{F}+R_{2}^{-1} \bar{M}\right) \bar{l}+R_{2}^{-1}\left((K+P+\bar{L}) \bar{x}^{(N)}+L \hat{x}+M \hat{l}+\varphi+\phi\right)\right] \mathrm{d} t, \quad \bar{l}(0)=0 . }
\end{aligned}\right.
$$

Remark 4.6. We use direct approach here and first obtain a set of centralizied optimal controls, then the decentralizied controls are designed. Note that $L, M, \bar{M}, \bar{L}, \varphi, \phi$ are not coupled with $\hat{x}$ and it is simpler to solve four Ricatti-liked equations than solving the CC system in [46] who contains five highly coupled FBSDEs. Thus, the fixed-point equation system is not necessary here. In addition, if $L, M, \bar{M}$ are symmetric, the original four equations can be even degenerated to three Ricatti-liked equations.

Remark 4.7. Note that here the weight $Q$ is allowed to be indefinite. If $Q$ is negative semi-definite, then equation (3.9) admits a solution necessarily. If $Q$ is positive semi-definite, then equation (4.14) also admits a solution necessarily. However, to ensure that both (3.9) and (4.14) admit solutions, the selection of $Q$ should reach a compromise and the magnitude of $\mathrm{Q}$ cannot be too "positive" or "negative".

\section{Asymptotic optimality}

Definition 5.1. A set of control laws $\bar{u}=\left\{\bar{u}_{1}, \bar{u}_{2}, \ldots, \bar{u}_{N}\right\} \in \mathcal{U}^{F}$ has robust asymptotic social optimality if

$$
\left|\frac{1}{N} \mathcal{J}_{\text {soc }}^{w o}(\bar{u})-\frac{1}{N} \inf _{u \in \mathcal{U}_{c}^{F}} \mathcal{J}_{\text {soc }}^{w o}(u)\right|=O\left(\frac{1}{\sqrt{N}}\right)
$$

where $\mathcal{U}_{c}^{F}$ is defined in Section 2 as a set of centralized information-based control.

Before proving asymptotically social optimality, we need to introduce some estimations first.

Lemma 5.2. Suppose that (A1)-(A6) hold. Then

$$
\mathbb{E} \int_{0}^{T}\left|\bar{x}^{(N)}-\hat{x}\right|^{2} \mathrm{~d} t+\mathbb{E} \int_{0}^{T}|\bar{l}-\hat{l}|^{2} \mathrm{~d} t=O\left(\frac{1}{N}\right) .
$$

Proof. By (4.19), we have

$$
\begin{aligned}
& \mathrm{d} \bar{x}^{(N)}=\left\{\left[A-B R^{-1} B^{T} K+\bar{F}-R_{2}^{-1} \bar{L}\right] \bar{x}^{(N)}-M\right) \hat{l}-B R_{1}^{-1} B^{T} L \hat{x}-B R_{1}^{-1} B^{T} \varphi+B R_{1}^{-1} B^{T}(P \\
& \left.-R_{2}^{-1}(\bar{M} \bar{l}+\phi)\right\} \mathrm{d} t+\frac{1}{N} \sum_{i=1}^{N} \sigma \mathrm{d} W_{i}, \quad \bar{x}^{(N)}(0)=\frac{1}{N} \sum_{i=1}^{N} \xi_{i} .
\end{aligned}
$$


Combining (4.10), (4.19), (4.17) and (5.1), one can obtain

$$
\left\{\begin{array}{l}
\mathrm{d} \tilde{x}=\left[\left(A-B R_{1}^{-1} B^{T} K+\bar{F}-R_{2}^{-1} \bar{L}\right) \tilde{x}-R_{2}^{-1} \bar{M} \tilde{l}\right] \mathrm{d} t+\frac{1}{N} \sum_{i=1}^{N} \sigma \mathrm{d} W_{i}, \quad \tilde{x}(0)=\frac{1}{N} \sum_{i=1}^{N} \xi_{i}-\hat{\xi} \\
\mathrm{d} \tilde{l}=\left[\left(A+\bar{F}-R_{2}^{-1} \bar{M}\right) \tilde{l}+R_{2}^{-1}(K+P+\bar{L}) \tilde{x}\right] \mathrm{d} t, \quad \tilde{l}(0)=0,
\end{array}\right.
$$

where $\tilde{x}=\bar{x}^{(N)}-\hat{x}, \tilde{l}=\bar{l}-\hat{l}$. By Cauchy-Schwarz inequality and Burkholder-Davis-Gundy's inequality, we have

$$
\begin{aligned}
\mathbb{E} \sup _{0 \leq s \leq t}|\mathbb{X}|^{2} & =\mathbb{E} \sup _{0 \leq s \leq t}\left|\mathbb{X}(0)+\int_{0}^{s} \mathbb{A} \mathbb{X} \mathrm{d} r+\int_{0}^{s} \frac{1}{N} \sum_{i=1}^{N}\left(\begin{array}{l}
\sigma \\
0
\end{array}\right) \mathrm{d} W_{i}\right|^{2} \\
& \leq c \mathbb{E} \sup _{0 \leq s \leq t} \int_{0}^{s}|\mathbb{X}|^{2} \mathrm{~d} r+\frac{3}{N^{2}} \sum_{i=1}^{N} \mathbb{E}\left|\int_{0}^{t}\left(\begin{array}{c}
\sigma \\
0
\end{array}\right)\right|^{2} \mathrm{~d} r \leq c \mathbb{E} \int_{0}^{t}|\mathbb{X}|^{2} \mathrm{~d} r+O\left(\frac{1}{N}\right),
\end{aligned}
$$

where $\mathbb{X}=\left(\tilde{x}^{T}, \tilde{l}^{T}\right)^{T}$,

$$
\mathbb{A}=\left(\begin{array}{cc}
A-B R_{1}^{-1} B^{T} K+\bar{F}-R_{2}^{-1} \bar{L} & -R_{2}^{-1} \bar{M} \\
R_{2}^{-1}(K+P+\bar{L}) & A+\bar{F}-R_{2}^{-1} \bar{M}
\end{array}\right),
$$

and constant $c$ is independent of $N$. Then, by Gronwall's inequality, one can obtain that

$$
\mathbb{E} \sup _{0 \leq t \leq T}|\mathbb{X}|^{2}=O\left(\frac{1}{N}\right)
$$

The lemma follows.

Remark 5.3. In [46], an additional Ricatti equation is needed for proving $\sup _{0 \leq t \leq T} \mathbb{E}\left(\left|\bar{x}^{(N)}-\hat{x}\right|^{2}+|\bar{s}-\hat{s}|^{2}\right)=$ $O\left(\frac{1}{N}\right)$, since $\left(\bar{x}^{(N)}-\hat{x}\right)$ and $(\bar{s}-\hat{s})$ satisfy a FBSDE system and they need to be decoupled by using Ricatti equation method. However, in our model, $\tilde{x}$ and $\tilde{l}$ evolve by forward SDEs and we can estimate them directly without setting such an assumption.

Theorem 5.4. Suppose that (A1)-(A6) hold. The set of decentralized control laws $\bar{u}(t)=\left\{\bar{u}_{1}(t), \bar{u}_{2}(t)\right.$, $\left.\ldots, \bar{u}_{N}(t)\right\} \in \mathcal{U}^{F}$ given by (4.18) has asymptotic social optimality.

Proof. Let $\grave{x}_{i}=x_{i}-\bar{x}_{i}, \grave{u}_{i}=u_{i}-\bar{u}_{i}, \grave{x}^{(N)}=x^{(N)}-\bar{x}^{(N)}$ and $\grave{s}=s-\bar{s}$, where $i=1,2, \ldots, N$. Then by (4.3),

$$
\left\{\begin{array}{l}
\left.\mathrm{d} \grave{x}_{i}=\left[A \grave{x}_{i}+B \grave{u}_{i}+\bar{F} \grave{x}^{(N)}-R_{2}^{-1} \grave{s}\right)\right] \mathrm{d} t, \quad \grave{x}_{i}(0)=0, \\
\mathrm{~d} \grave{s}=-\left[(A+\bar{F})^{T} \grave{s}-P B \grave{u}^{(N)}\right] \mathrm{d} t+\frac{1}{N} \sum_{j=1}^{N} \sum_{i=1}^{N}\left(-\grave{\beta}_{i}^{j}\right) \mathrm{d} W_{j}, \quad \grave{s}(T)=0 .
\end{array}\right.
$$

By Lemma 5.4 in [27], if (A1)-(A6) hold, for all $u_{i} \in \mathcal{U}_{c}^{F}, i=1,2, \ldots, N$, we have $\frac{1}{N} \mathcal{J}_{\text {soc }}^{\text {wo }}(u) \leq \frac{1}{N} \mathcal{J}_{\text {soc }}^{\text {wo }}(\bar{u}) \leq c$, where $c$ is independent of $N$. That implies $\mathbb{E} \int_{0}^{T}\left|u_{i}\right|^{2} \mathrm{~d} t<c$. Then, by (5.3), we have $\mathbb{E} \int_{0}^{T}\left(\left|\grave{x}_{i}\right|^{2}+\left|\grave{u}_{i}\right|^{2}+|\grave{s}|^{2}\right) \mathrm{d} t<$ 
c. Next, considering (2.2) and (4.2), we denote

$$
\begin{aligned}
\mathcal{J}_{\text {soc }}^{F}(u)= & \frac{1}{2} \sum_{i=1}^{N} \mathbb{E} \int_{0}^{T}\left\{\left|x_{i}-\Gamma x^{(N)}-\eta\right|_{Q}^{2}+\left|u_{i}\right|_{R_{1}}^{2}-\left|P x^{(N)}+s\right|_{R_{2}^{-1}}^{2}\right\} \mathrm{d} t \\
& +\frac{1}{2} \sum_{i=1}^{N} \mathbb{E}\left|x_{i}(T)-\hat{\Gamma} x^{(N)}(T)-\hat{\eta}\right|_{G}^{2}=\sum_{i=1}^{N}\left(\mathcal{J}_{i}^{F}(\bar{u})+\grave{\mathcal{J}}_{i}^{F}(\grave{u})+I_{i}\right)
\end{aligned}
$$

where

$$
\grave{J}_{i}^{F}(\grave{u})=\frac{1}{2} \mathbb{E} \int_{0}^{T}\left\{\left|\grave{x}_{i}-\Gamma \grave{x}^{(N)}\right|_{Q}^{2}+\left|\grave{u}_{i}\right|_{R_{1}}^{2}-\mid P \grave{x}^{(N)}+\grave{s}_{R_{2}^{-1}}^{2}\right\} \mathrm{d} t+\frac{1}{2} \mathbb{E}\left|\grave{x}_{i}(T)-\hat{\Gamma} \grave{x}^{(N)}(T)\right|_{G}^{2}
$$

and

$$
\begin{aligned}
\sum_{i=1}^{N} I_{i}= & \sum_{i=1}^{N} \mathbb{E} \int_{0}^{T}\left\langle Q \bar{x}_{i}-Q_{\Gamma} \bar{x}^{(N)}-\eta_{\Gamma}-P R_{2}^{-1}(P \hat{x}+\hat{s}), \grave{x}_{i}\right\rangle-\left\langle P R_{2}^{-1}\left[P\left(\bar{x}^{(N)}-\hat{x}\right)+(\bar{s}-\hat{s})\right], \grave{x}_{i}\right\rangle \\
& -\left\langle R_{2}^{-1}(P \hat{x}+\hat{s}), \grave{s}\right\rangle-\left\langle R_{2}^{-1}\left[P\left(\bar{x}^{(N)}-\hat{x}\right)+(\bar{s}-\hat{s})\right], \grave{s}\right\rangle+\left\langle R_{1} \bar{u}_{i}, \grave{u}_{i}\right\rangle \mathrm{d} t \\
& +\sum_{i=1}^{N} \mathbb{E}\left\langle G \bar{x}_{i}(T)-G_{\hat{\Gamma}} \bar{x}^{(N)}(T)-\hat{\eta}_{\hat{\Gamma}}, \grave{x}_{i}(T)\right\rangle .
\end{aligned}
$$

We now prove $\sum_{i=1}^{N} I_{i}=O\left(\frac{1}{\sqrt{N}}\right)$. According to the discussion in the last section, it follows that

$$
\bar{k}_{i}=K \bar{x}_{i}+L \hat{x}+M \hat{l}+\varphi, \quad \bar{k}_{i}(T)=G \bar{x}_{i}(T)-G_{\hat{\Gamma}} \bar{x}^{(N)}(T)-\hat{\eta}_{\hat{\Gamma}} .
$$

By (4.19), (5.3) and the similar techniques from (4.4) to (4.9),

$$
\begin{aligned}
\sum_{i=1}^{N} \mathbb{E}\left\langle\bar{k}_{i}(T), \grave{x}_{i}(T)\right\rangle= & \sum_{i=1}^{N} \mathbb{E} \int_{0}^{T}\left\langle-Q \bar{x}_{i}+Q_{\Gamma} \bar{x}^{(N)}+\eta_{\Gamma}-K R_{2}^{-1}(\bar{s}-\hat{s})+P R_{2}^{-1}(P+\bar{L}) \hat{x}+P R_{2}^{-1} \bar{M} \hat{l}\right. \\
& \left.+P R_{2}^{-1} \phi+\left(K \bar{F}+\bar{F}^{T} K\right)\left(\bar{x}^{(N)}-\hat{x}\right), \grave{x}_{i}\right\rangle-\left\langle R_{2}^{-1}\left(K \bar{x}_{i}+L \hat{x}+M \hat{l}+\varphi\right), \grave{s}\right\rangle \\
& +\left\langle B^{T}\left(K \bar{x}_{i}+L \hat{x}+M \hat{l}+\varphi\right), \grave{u}_{i}\right\rangle \mathrm{d} t,
\end{aligned}
$$

and

$$
\begin{aligned}
0= & \sum_{i=1}^{N}\langle\bar{l}(T), \grave{s}(T)\rangle-\langle\bar{l}(0), \grave{s}(0)\rangle=\sum_{i=1}^{N} \mathbb{E} \int_{0}^{T}\left\langle R_{2}^{-1} \bar{k}_{i}, \grave{s}\right\rangle+\left\langle R_{2}^{-1}(P \hat{x}+\hat{s}), \grave{s}\right\rangle-\left\langle B^{T} P \bar{l}, \grave{u}_{i}\right\rangle \\
& +\left\langle R_{2}^{-1}(P+\bar{L})\left(\bar{x}^{(N)}-\hat{x}\right)+R_{2}^{-1} \bar{M}(\bar{l}-\hat{l}), \grave{s}\right\rangle .
\end{aligned}
$$

Combining (5.5)-(5.8) and Lemma 5.2, note that $\bar{s}-\hat{s}=\bar{M} \tilde{l}+\bar{L} \tilde{x}$ and $R_{1} \bar{u}_{i}+B^{T}\left[K \bar{x}_{i}+L \hat{x}-B^{T}(P-M) \hat{l}+\varphi\right]=$ 0 , one can obtain

$$
\frac{1}{N} \sum_{i=1}^{N} I_{i}=O\left(\frac{1}{\sqrt{N}}\right) .
$$

Then, we put this into (5.4), the theorem follows. 


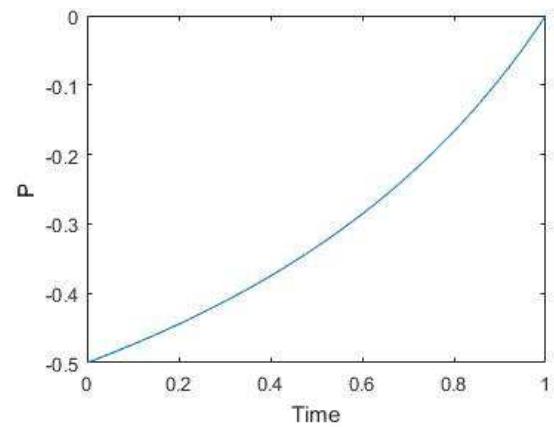

(a)

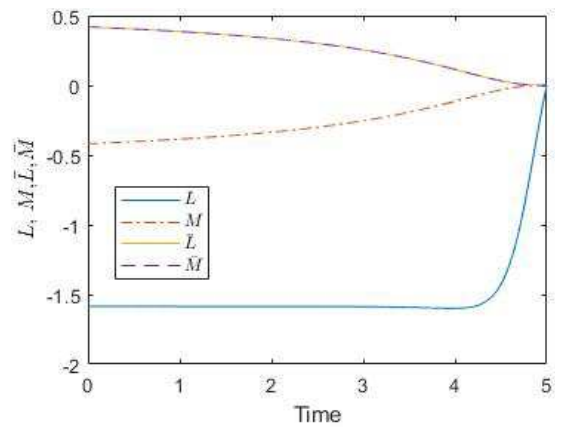

(b)

Figure 1. (a) is the curve of $P(t)$ and (b) is the curves of $L, M, \bar{L}$ and $\bar{M}$.

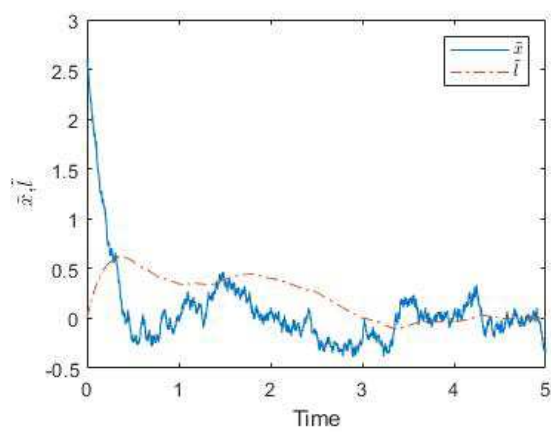

(a)

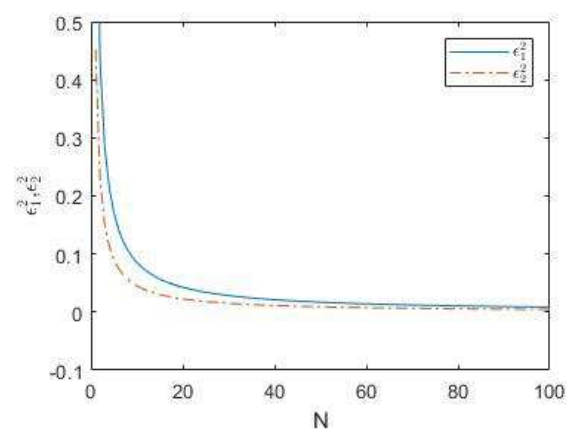

(b)

Figure 2. (a) is the curves of $\tilde{x}, \tilde{l}$ and (b) is the curves of $\varepsilon_{1}^{2}, \varepsilon_{2}^{2}$ when time interval is $[0,5]$.

\section{Numerical EXAMPLES}

We continue to use the parameters in Example 3.2. First, we give the figure of $P(t)=-\frac{1}{t-2}-1, t \in[0,1]$. Since $P(t)$ in (3.2) is the same as it in (3.9), $P(t)=-\frac{1}{t-2}-1$ could also be solution for (3.9) and its trajectory is shown in Figure 1a. Let the population $N=100, R_{1}=0.5, \sigma=5, \eta=\hat{\eta}=0, \hat{\Gamma}=0.5$ and the time interval is $[0,5]$. Using Matlab computation and by (3.9), (4.14), $P$ and $K$ can be easily computed. After that, we simulate the BSDEs from (4.15) to (4.16) and obtain their figures in Figure $1 \mathrm{~b}^{1}$. Taking the initial values independently from a uniform distribution $U(-30,60)$ and by equations $(5.2)$, the curve of $\tilde{x}, \tilde{l}$ and $\tilde{s}$ is described in Figure $2 \mathrm{a}$. Denote that $\varepsilon_{1}^{2}=\mathbb{E} \int_{0}^{1}\left|\bar{x}^{(N)}-\hat{x}\right|^{2} \mathrm{~d} t, \varepsilon_{2}^{2}=\mathbb{E} \int_{0}^{1}|\bar{l}-\hat{l}|^{2} \mathrm{~d} t$. We let $N$ increase from 1 to 100 and the curves of $\varepsilon_{1}^{2}$ and $\varepsilon_{2}^{2}$ are shown in Figure 2b. It shows that they are getting close to zero when $N$ is becoming larger and larger.

\section{Conclusion}

This paper considered a social optimality for robust LQ-MF problem with a common uncertain drift. By the robust optimization approach, we obtain a "worst case" disturbance for all agents. Using variational analysis and decoupling FBSDEs with mean field approximation, we construct the decentralized controls, which are

\footnotetext{
${ }^{1}$ By observing Figure 1b, we could find that the curve of $\bar{L}$ and $\bar{M}$ is overlapping. In fact, the situation does not change even though we try many sets of numbers for the parameters. Therefore, we have a hypothesis that $\bar{L}$ may be equal to $\bar{M}$. If so, the system (4.15) and (4.16) are decoupled and solved directly, which may be useful in other more complicated models. Unfortunately, we cannot prove the hypothesis rigorously in mathematics.
} 
further proved to be an asymptotically social optimum. For further work, it is interesting to investigate social optimality for robust mean field LQ problem with uncertainty in common noise by the direct approach.

\section{REFERENCES}

[1] A. Aurell and B. Djehiche, Mean-field type modeling of nonlocal crowd aversion in pedestrian crowd dynamics. SIAM J. Control Optim. 56 (2018) 434-455.

[2] A. Aurell, R. Carmona, G. Dayanikli and M. Lauriere, Optimal incentives to mitigate epidemics: a Stackelberg mean field game approach. Preprint arXiv:2011.03105v1 (2020).

[3] M. Bardi and F.S. Priuli, Linear-quadratic N-person and mean-field games with ergodic cost. SIAM J. Control Optim. 52 (2014) 3022-3052.

[4] T. Başar and P. Bernhard, $H^{\infty}$-optimal Control and Related Minimax Design Problems: A Dynamic Game Approach, 2nd ed. Birkhauser, Boston, MA (1995).

[5] C.T. Bauch and D.J.D. Earn, Vaccination and the theory of games. P. Natl. Acad. Sci. 101 (2004) 13391-13394.

[6] A. Bensoussan, J. Frehse and P. Yam, Mean Field Games and Mean Field Type Control Theory. Springer, New York (2013).

[7] W.A. van den Broek, J.C. Engwerda and J.M. Schumacher, Robust equilibria in indefinite linear-quadratic differential games. J. Optim. Theory Appl. 119 (2003) 565-595.

[8] M. Burger, M.D. Francesco, P.A. Markowich and M.T. Wolfram, Mean field games with nonlinear mobilities in pedestrian dynamics. Discrete Continuous Dyn. Syst. Ser. B 19 (2014) 1311-1333.

[9] P.E. Caines, Mean field games, in Encyclopedia of Systems and Control, edited by T. Samad and J. Baillieul. Springer-Verlag, Berlin (2014).

[10] R. Carmona and F. Delarue, Probabilistic analysis of mean-field games. SIAM J. Control Optim. 51 (2013) $2705-2734$.

[11] R. Carmona and F. Delarue, Probabilistic Theory of Mean Field Games with Applications I-II. Springer (2018).

[12] R. Carmona and P. Wang, Finite-state contract theory with a principal and a field of agents. Preprint arXiv:1808.07942 (2018).

[13] P. Chan and R. Sircar, Fracking, renewables, and mean field games. SIAM Rev. 59 (2017) 588-615.

[14] S. Cho, Mean-field game analysis of SIR model with social distancing. Preprint arXiv:2005.06758 (2020).

[15] R. Couillet, S.M. Perlaza, H. Tembine and M. Debbah, Electrical vehicles in the smart grid: a mean field game analysis. IEEE J. Sel. Area. Commun. 30 (2012) 1086-1096.

[16] T.E. Duncan and H. Tembine, Linear-quadratic mean-field-type games: a direct method. Games 9 (2018) 7.

[17] R. Elie, E. Hubert and G. Turinici, Contact rate epidemic control of COVID-19: an equilibrium view. Preprint arXiv:2004.08221 (2020).

[18] J. Engwerda, A numerical algorithm to find soft-constrained Nash equilibria in scalar LQ-games. Int. J. Control 79 (2006) 592-603.

[19] D. Firoozi and P.E. Caines, Mean field game $\varepsilon$-Nash equilibria for partially observed optimal execution problems in finance. Proc. the IEEE 55th Conference on Decision and Control (2016) 268-275.

[20] G. Freiling, A survey of nonsymmetric Riccati equations. Linear Algebra Appl. 351 (2002) 243-270.

[21] B. Gaujal, J. Doncel and N. Gast, Vaccination in a Large Population: Mean Field Equilibrium versus Social Optimum. In netgcoop'20.

[22] G. Gnecco, M. Sanguineti and M. Gaggero, Suboptimal solutions to team optimization problems with stochastic information structure. SIAM J. Control Optim. 22 (2012) 212-243.

[23] Y.C. Ho and K.C. Chu, Team decision theory and information structures in optimal control Part I. IEEE Trans. Automat. Control 17 (1972) 15-22.

[24] E. Hubert and G. Turinici, Nash-MFG equilibrium in a SIR model with time dependent newborn vaccination. Ric. di Mat. 67 (2018) 227-246.

[25] J. Huang and M. Huang, Mean field LQG games with model uncertainty. Proc. 52nd IEEE International Conference on Decision and Control (2013) 3103-3108.

[26] J. Huang and M. Huang, Robust mean field linear-quadratic-Gaussian games with unknown $L^{2}$-disturbance. SIAM J. Control Optim. 55 (2017) 2811-2840.

[27] J. Huang, B. Wang and T. Xie, Social optima in leader-follower mean field linear quadratic control. ESAIM: COCV 27 (2021) $\mathrm{S} 12$.

[28] M. Huang, P.E. Caines and R.P. Malhamé, Individual and mass behaviour in large population stochastic wireless power control problems: centralized and Nash equilibrium solutions. Proc. 42nd IEEE International Conference on Decision and Control (2003) 98-103.

[29] M. Huang, P.E. Caines and R.P. Malhamé, Social optima in mean-field LQG control: centralized and decentralized strategies. IEEE Trans. Automat. Contr. 57 (2012) 1736-1751.

[30] M. Huang and M. Zhou, Linear-quadratic mean field games: asymptotic solvability and relation to the fixed point approach. IEEE Trans. Automat. Contr. 65 (2020) 1397-1412.

[31] A.C. Kizilkale and R.P. Malhame, Collective target tracking mean field control for markovian jump-driven models of electric water heating loads. Proc. 19th IFAC World Congress, Cape Town, South Africa (2014) 1867-1972.

[32] D. Kremer and R. Stefan, Non-symmetric Riccati theory an linear quadratic Nash games. Proc. 15th Internat. Symp. Math. Theory Networks and Systems (MTNS), Univ, Notre Dame, USA (2014). 
[33] A. Lachapelle and M.T. Wolfram, On a mean field game approach modeling congestion and aversion in pedestrian crowds. Transport Res. B 45 (2011) 1572-1589.

[34] J. Lasry and P. Lions, Mean field games. Jpn. J. Math. 2 (2007) 229-260.

[35] L. Laguzet and G. Turinici, Individual vaccination as Nash equilibrium in a SIR model with application to the 2009-2010 influenza A (H1N1) epidemic in France. Bull. Math. Biol. 77 (2015) 1955-1984.

[36] T. Li and J. Zhang, Asymptotically optimal decentralized control for large population stochastic multiagent systems. IEEE Trans. Automat. Contr. 53 (2008) 1643-1660.

[37] J. Ma and J. Yong, Forward-backward Stochastic Differential Equations and their Applications, Lecture Notes in Math. Springer-Verlag (1999).

[38] J. Moon and T. Başar, Linear quadratic risk-sensitive and robust mean field games. IEEE Trans. Automat. Contr. 62 (2017) $1062-1077$.

[39] H. Strube, Time-varying wave digital filters and vocal-tract models. IEEE International Conference on Acoustics, Speech, and Signal Processing (1982) 923-926.

[40] J. Sun, X. Li and J. Yong, Open-loop and closed-loop solvabilities for stochastic linear quadratic optimal control problems. SIAM J. Control Optim. 54 (2016) 2274-2308.

[41] T. Sung, S. Yoon and K. Kim, A mathematical model of hourly solar radiation in varying weather conditions for a dynamic simulation of the solar organic Rankine cycle. Energies 8 (2015) 7058-7069.

[42] H. Tembine, D. Bauso and T. Başar, Robust linear quadratic mean-field games in crowd-seeking social networks. Proc. 52nd IEEE International Conference on Decision and Control (2013) 3134-3139.

[43] B. Wang and J. Zhang, Mean field games for large population multiagent systems with Markov jump parameters. SIAM J. Control Optim. 50 (2012) 2308-2334.

[44] B. Wang and J. Zhang. Social optima in mean field linear-quadratic-Gaussian models with Markov jump parameters. SIAM J. Control Optim. 55 (2017) 429-456.

[45] B. Wang and J. Huang, Social optima in robust mean field LQG control. The 11th Asian Control Conference (ASCC), Gold Coast, QLD (2017) 2089-2094.

[46] B. Wang, J. Huang and J. Zhang, Social optima in robust mean field LQG control: from finite to infinite horizon. IEEE Trans. Automat. Contr. 57 (2012) 1736-1751.

[47] B. Wang and M. Huang, Mean field production output control with sticky prices: Nash and social solutions. Automatica 100 (2019) 590-598.

[48] B. Wang, H. Zhang and J. Zhang, Mean field linear quadratic control: uniform stabilization and social optimality. Automatica $121(2020) 109088$

[49] G.Y. Weintraub, C.L. Benkard and B.V. Roy, Markov perfect industry dynamics with many firms. Econometrica 76 (2008) $1375-1411$.

[50] J. Yong and X.Y. Zhou, Stochastic Controls: Hamiltonian Systems and HJB Equations. Springer-Verlag (1999). 